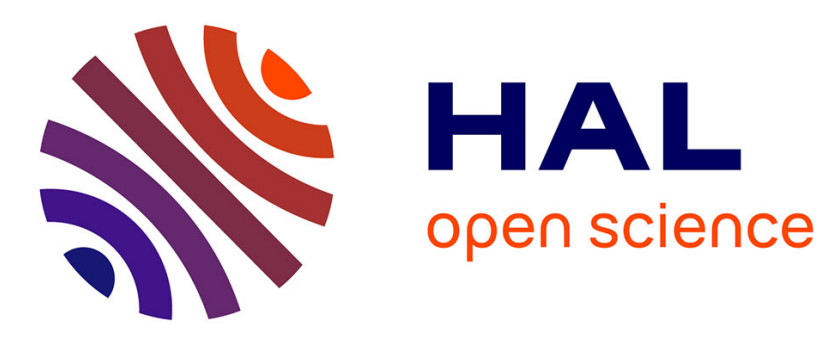

\title{
Propriétés d'une structure à auges utilisable dans un accélérateur linéaire supraconducteur
}

\author{
M. Boussoukaya, L. Wartski, A. Septier
}

\section{To cite this version:}

M. Boussoukaya, L. Wartski, A. Septier. Propriétés d'une structure à auges utilisable dans un accélérateur linéaire supraconducteur. Revue de Physique Appliquée, 1978, 13 (3), pp.125-134. 10.1051/rphysap:01978001303012500 . jpa-00244430

\section{HAL Id: jpa-00244430 https://hal.science/jpa-00244430}

Submitted on 1 Jan 1978

HAL is a multi-disciplinary open access archive for the deposit and dissemination of scientific research documents, whether they are published or not. The documents may come from teaching and research institutions in France or abroad, or from public or private research centers.
L'archive ouverte pluridisciplinaire HAL, est destinée au dépôt et à la diffusion de documents scientifiques de niveau recherche, publiés ou non, émanant des établissements d'enseignement et de recherche français ou étrangers, des laboratoires publics ou privés. 
Classification

Physics Abstracts

06.71

\title{
PROPRIÉTÉS D'UNE STRUCTURE A AUGES UTILISABLE DANS UN ACCÉLÉRATEUR LINÉAIRE SUPRACONDUCTEUR
}

\author{
M. BOUSSOUKAYA, L. WARTSKI \\ Institut d'Electronique Fondamentale, Laboratoire associé au C. N. R. S., \\ Université Paris-XI, Bâtiment 220, 91405 Orsay Cedex, France
}

et

\section{A. SEPTIER}

C. N. A. M., 292, rue Saint-Martin, 75141 Paris Cedex 03, France

(Reçu le 19 septembre 1977, révisé le 21 novembre 1977 et accepté le 6 décembre 1977)

\begin{abstract}
Résumé. - Nous étudions expérimentalement les propriétés, à la température ambiante, de modèles de structures accélératrices à symétrie plane : les structures à auges. Les caractéristiques de dispersion, ainsi que les paramètres importants : $Q_{0}, R_{\text {eff }} / Q_{0}, E_{\mathrm{p}} / E_{\text {eff }}, B_{\mathrm{p}} / E_{\text {eff, }}$ ont été déterminés expérimentalement pour trois géométries différentes en fonction de l'écartement entre les plaques-supports des auges et pour un fonctionnement en mode $\pi$ ou $\pi / 2$. Les résultats les plus intéressants pour une utilisation dans un accélérateur supraconducteur ont été obtenus pour une structure utilisant des parois inclinées et des bords arrondis.
\end{abstract}

\begin{abstract}
We investigate experimentally the properties at room temperature of different models of the so-called muffin-tin accelerating structure. The dispersion curves as well as the important parameters : $Q_{0}, R_{\text {eff }} / Q_{0}, E_{\mathrm{p}} / E_{\text {eff }}, B_{\mathrm{p}} / E_{\text {eff }}$ have been measured for three different geometries as a function of the spacing of the plates supporting the periodic structure and for the $\pi$ or $\pi / 2$ mode. The best results for the use in a superconducting accelerator are obtained in a structure in which the walls separating the cells are slightly inclined and the edges rounded.
\end{abstract}

1. Introduction. - Une étude systématique des propriétés de structures à ondes lentes à symétrie plane - du type guide à auges [1-4] - effectuée sur cavités résonnantes a permis d'identifier différents modes accélérateurs, d'en tracer les courbes de dispersion, et de déterminer les valeurs de l'impédance shunt correspondantes. La méthode des perturbations a fourni les valeurs de deux paramètres très importants lorsqu'on envisage l'utilisation de ces structures dans un accélérateur linéaire supraconducteur : les rapports du champ électrique et du champ magnétique maximums sur la paroi au champ accélérateur sur l'axe.

Trois structures ont été étudiées. Deux sont à auges de section rectangulaire ayant pour le mode $\pi$, l'une une vitesse de phase $v_{\varphi}$ telle que $v_{\varphi} \simeq c$, l'autre $v_{\varphi} \simeq c / 2$. La troisième, qui comporte des auges de section trapézoïdale à bords arrondis, dans laquelle $v_{\varphi} \simeq c$ pour le mode $\pi$, permet d'obtenir, à impédance shunt égale, des champs sur la paroi plus faibles que dans les deux autres structures, et paraît donc mieux adaptée à la réalisation d'un guide supraconducteur. Après une description des structures et des méthodes de mesure, nous donnons les résultats expérimentaux obtenus.
2. Structures accélératrices à auges à symétrie plane. - Toutes ces structures sont obtenues en plaçant face à face, à la distance $e$, une série d'auges identiques creusées par fraisage dans des plaques métalliques épaisses et séparées par des parois d'épaisseur $t$. Le pas de la structure correspond, pour le mode $\pi$ à $p=\lambda_{0} / 2$ dans le cas où l'on désire obtenir $v_{\varphi}=c$, et à $p=\lambda_{0} / 4$ lorsque $v_{\varphi}=c / 2$. L'écartement est assuré par deux barres métalliques d'épaisseur $e$ placées aux extrémités, parallèlement à l'axe $0 x$, et à une distance $d$ du bord des auges terminales (Fig. 1). La cavité résonnante ainsi formée, n'est, en général, pas fermée sur ses faces latérales, normales à $O x$, une étude préliminaire [5] ayant montré que les pertes sont plus faibles lorsque les ondes qui se propagent suivant $\mathrm{O} z$ se raccordent, dans la zone latérale d'épaisseur $e$, à des champs évanescents suivant $O x$. Toutefois, dans plusieurs séries de mesures des barres d'épaisseur $e$ ont été placées normalement à l'axe $\mathrm{O} x$, à la distance $D$ du bord des auges ( $D$ peut varier de 0 à $30 \mathrm{~mm}$, et l'absence de barres correspond à $D$ infini).

Le choix de la distance $d$ des plans de court-circuit au bord des auges terminales résulte de considérations analogues. Dans les modèles que nous avons étudiés, 


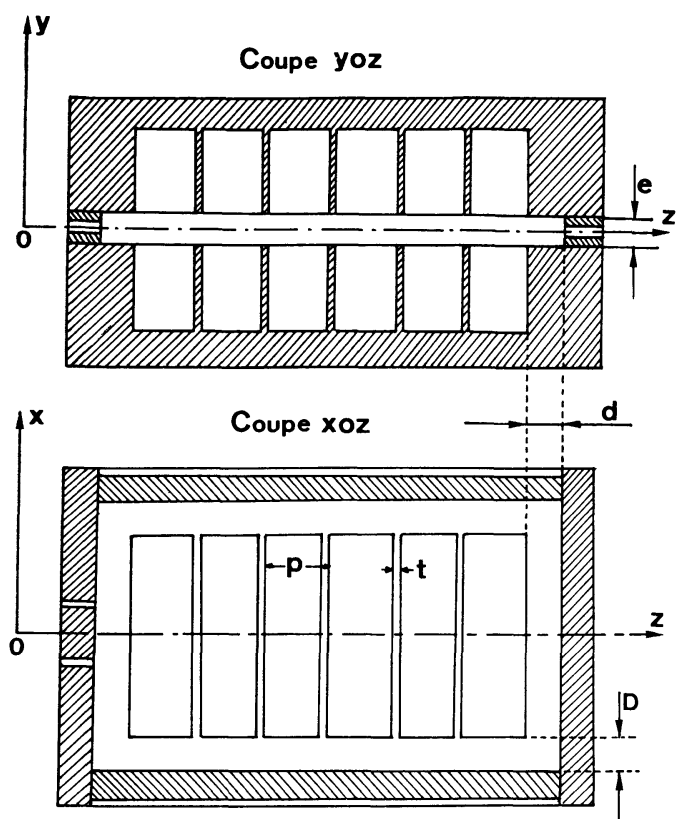

Fig. 1. - Schéma de la cavité parallélépipédique à auges. Notations.

[Schematic diagram of the muffin tin structure. Notations used.]

qui, par commodité de construction, sont tous terminés par des auges entières, l'excitation du mode $\pi$ ne peut généralement être observée que si les plans de courtcircuit sont situés en dehors des plans de symétrie de la structure (plans médians des parois de séparation des auges). Nous avons obtenu une amélioration notable du coefficient de surtension en prenant $d \geqslant 7,5 \mathrm{~mm}$.

Deux antennes courtes reliées à des lignes coaxiales, placées dans l'une des barres terminales assurent, l'une l'excitation des champs électromagnétiques dans la cavité, l'autre la détection de ces champs lors des résonances.

Trois structures à auges ont été étudiées successivement. La première qui comporte $2 \times 6$ auges a été réalisée en laiton; les deux autres, à $2 \times 4$ auges, en cuivre OFHC. Pour toutes les structures, on a fait varier l'écartement $e$ entre les séries d'auges.

La première structure, identique à l'une de celles étudiées à Cornell [1-3] comporte des auges parallélépipédiques de $76 \times 47,5 \times 25 \mathrm{~mm}^{3}$ séparées par des parois planes à bord arrondi d'épaisseur $t=5 \mathrm{~mm}$. Pour le mode $\pi$ du mode accélérateur fondamental la vitesse de phase $v_{\varphi}$ des ondes directe et réfléchie est proche de $c$, vitesse de la lumière. Dans la seconde structure, également à auges de section rectangulaire $\left(60 \times 25 \times 20 \mathrm{~mm}^{3}\right.$ avec parois de $\left.5 \mathrm{~mm}\right)$, on a cherché à obtenir $v_{\varphi} \simeq c / 2$ pour le mode $\pi$. Enfin, dans la troisième, un profil d'auge différent (Fig. $2 a$ et $2 b$ ) a été retenu, pour réduire les valeurs des champs électromagnétiques $E_{\mathrm{p}}$ et $B_{\mathrm{p}}$ sur les parois.

3. Courbes de dispersion des modes accélérateursbandes passantes et coefficients de surtension. L'identification des différents modes de résonance ne

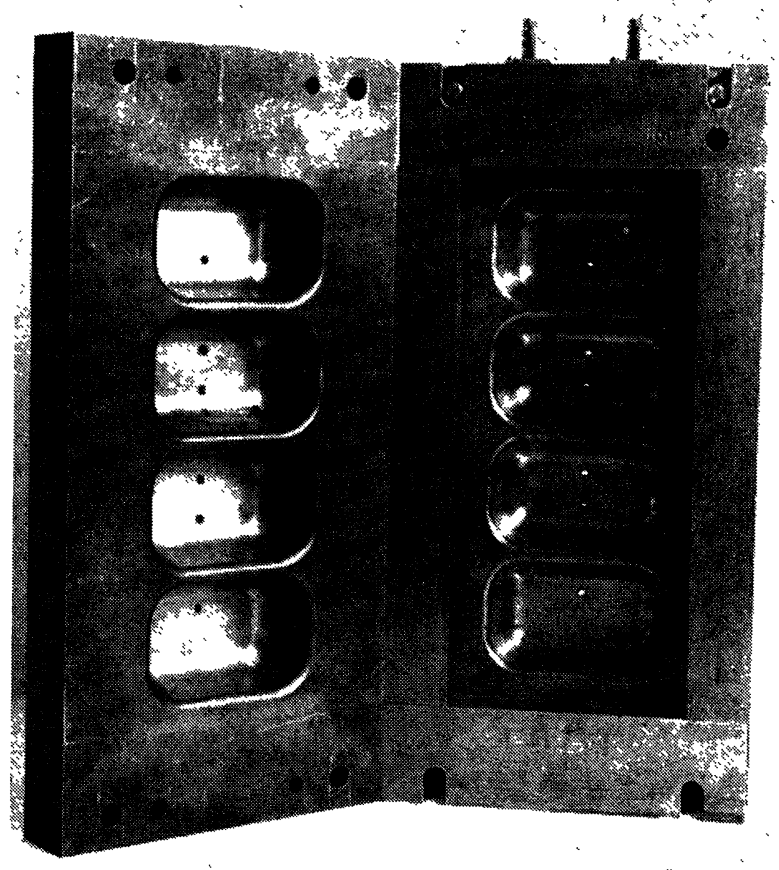

FIG. 2a. - Photographie des deux plaques constituant la cavité à auges trapézoïdales à bords arrondis (cavité $n^{\circ} 3$ ).

[Photograph of the two halves of the modified muffin tin structure.]

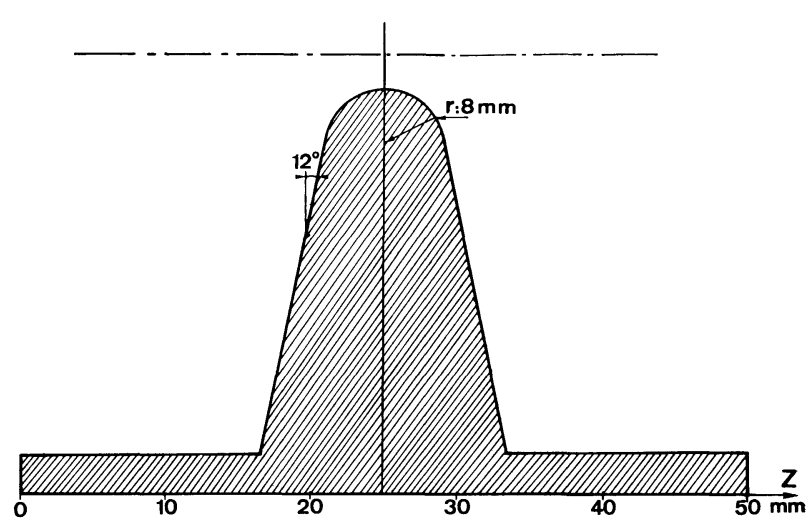

FIG. 2b. - Coupe $y \mathrm{O} z$ montrant le profil des auges dans la cavité $n^{0} 3$.

[ $y \mathrm{O} z$ cross-section showing the muffin tin profile in cavity $\left.\mathrm{n}^{\circ} 3.\right]$

peut être obtenue avec certitude qu'en relevant la courbe de variation suivant $\mathrm{O} z$ de l'amplitude $E_{z}$ du champ accélérateur. Pour cela, nous avons utilisé une très courte aiguille diélectrique, sensible seulement au champ $E_{z}$, se déplaçant sur l'axe de la structure.

Cette identification a été effectuée, par continuité, en partant de la structure dans laquelle les plans de court-circuit sont placés dans une position correspondant à des plans de symétrie des parois $(d=2,5 \mathrm{~mm})$ simulant un guide de longueur infinie. Une amélioration a ainsi été apportée aux résultats présentés précédemment [5], grâce à cette méthode de lever de doute.

La figure $3 a-f$ montre à titre d'exemple les répartitions de $E_{z}^{2}(z)$ enregistrées aux différentes fréquences de 


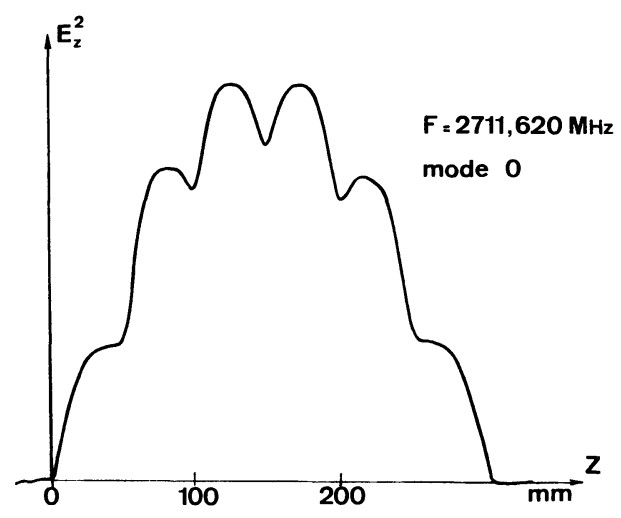

(a)

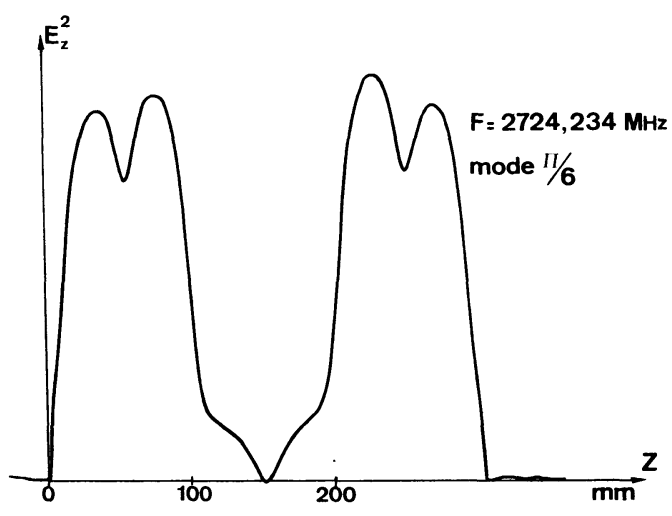

(b)

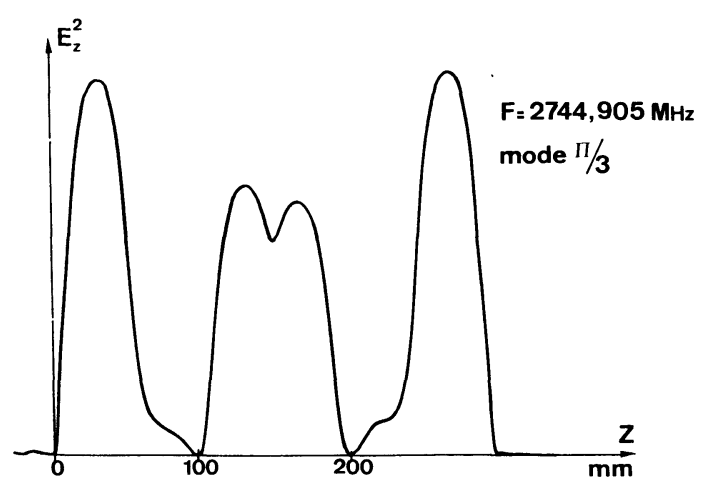

(e)

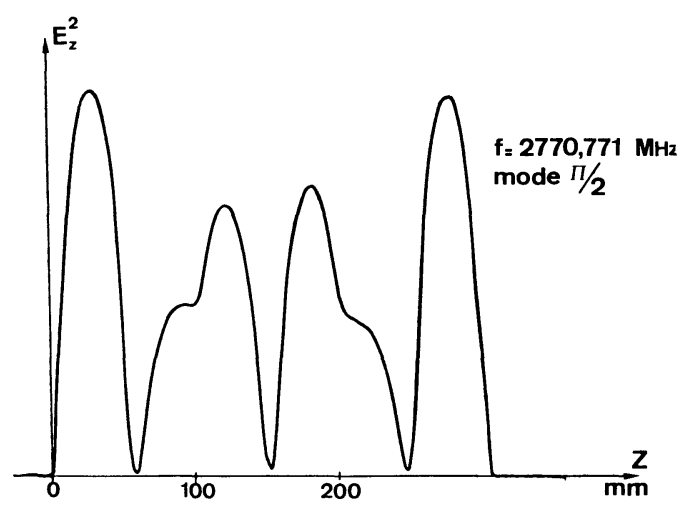

(d)

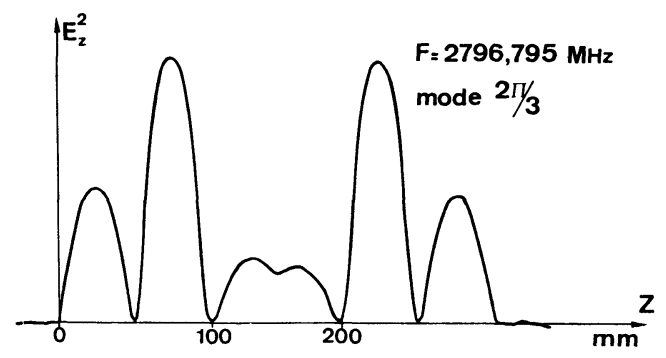

(e)
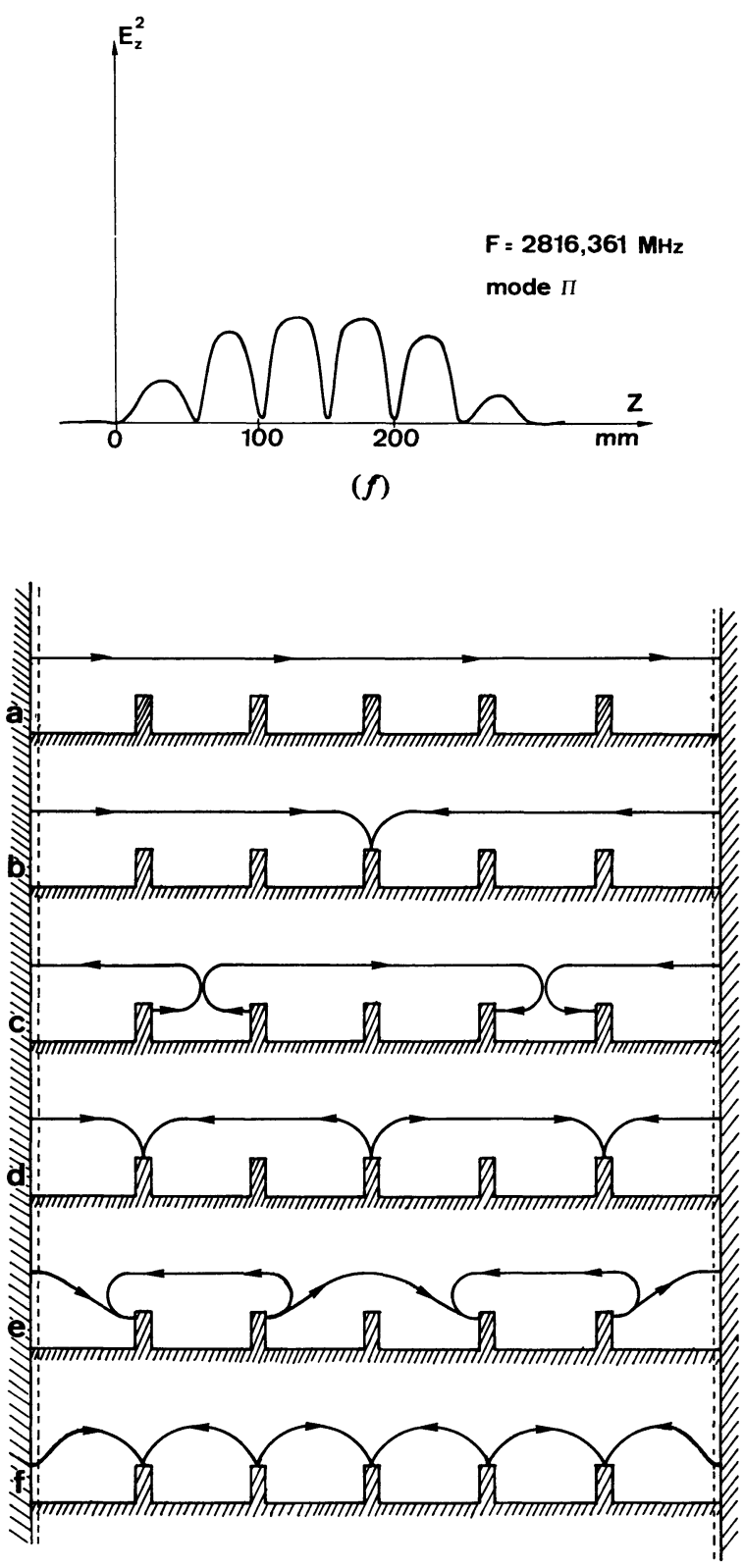

(g)

FIG. 3. - Exemples de distribution de $E_{\hat{z}}^{\hat{\imath}}(z)$ enregistrées aux différentes fréquences de résonance de la cavité $n^{\circ} 1$ (unités arbitraires).

[Examples of the $E_{z}^{2}(z)$ distribution at different resonance frequencies in cavity $n^{\circ} 1$ (arbitrary units).] 
résonance de la cavité $\mathrm{n}^{\circ} 1$ à 6 auges lorsque $e=25 \mathrm{~mm}$ et $d=7,5 \mathrm{~mm}$. On notera, outre la présence des harmoniques d'espace, une diminution importante de $E_{z}$ dans les auges terminales à la fréquence de résonance du mode $\pi$.

On a représenté schématiquement sur la figure $3 g$ l'allure des lignes de champ électrique correspondant à la nomenclature des modes ainsi identifiés. Le mode $\pi$ peut être excité grâce à l'existence de champs évanescents. Il ne pourrait pas l'être dans la structure avec plans réfléchissants placés dans les plans de symétrie des parois (lignes pointillées).

La figure 4 donne les courbes de dispersion relevées dans les trois structures pour le mode accélérateur fon-

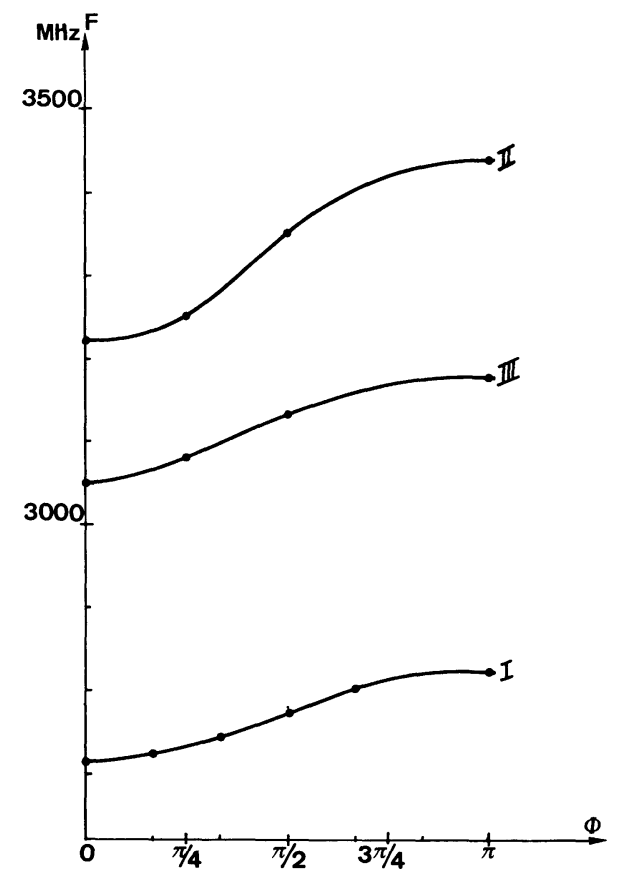

Fig. 4. - Caractéristiques de dispersion du mode accélérateur fondamental pour les trois modèles de structures étudiées : $e=25 \mathrm{~mm} ; d=7,5 \mathrm{~mm} ; D=\infty$.

[Dispersion curves of the fundamental accelerating mode for the three models of muffin tin structures. $e=25 \mathrm{~mm} ; d=7,5 \mathrm{~mm}$;

$$
D=\infty \text {. }
$$

damental, avec $e=25 \mathrm{~mm}$. Nous avons reporté sur la figure 5 l'évolution de la bande passante de chaque structure définie par :

$$
\gamma=2 \frac{f_{\pi}-f_{0}}{f_{\pi}+f_{0}}
$$

en fonction de l'écartement $e$. La figure 6 montre l'évolution de la fréquence du mode $\pi$ en fonction de ce même écartement.

Les coefficients de surtension $Q_{0}$ obtenus dans la structure $\mathrm{n}^{0} 3$ à 4 auges réalisée en cuivre OFHC sont données sur le tableau I en fonction de l'écartement $e$ pour le mode $\pi$, pour lequel la vitesse de phase d'une onde progressive serait voisine de $c$. Dans ce cas, la

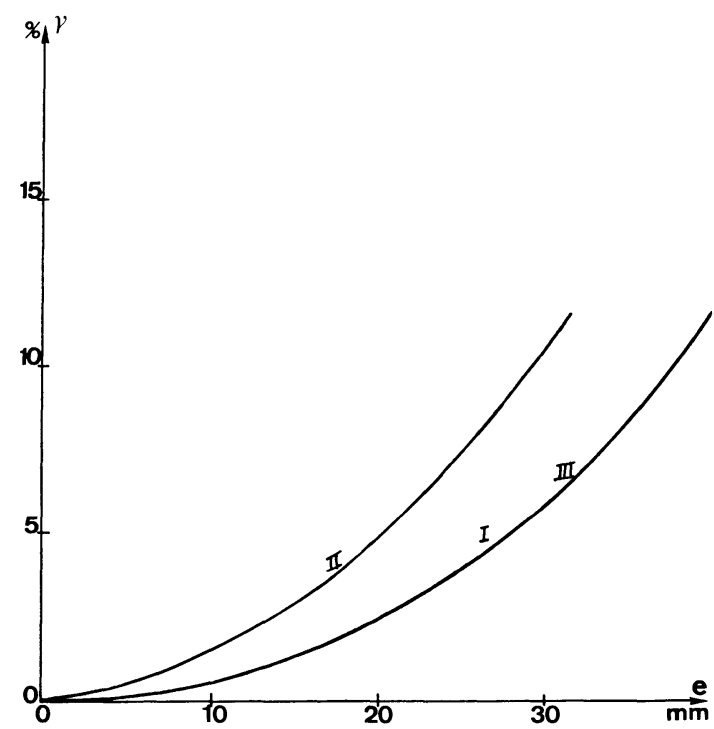

Fig. 5. - Evolution de la bande passante en fonction de l'écartement des plaques $e$.

[Evolution of the bandwidth versus the spacing $e$ of the plates.]

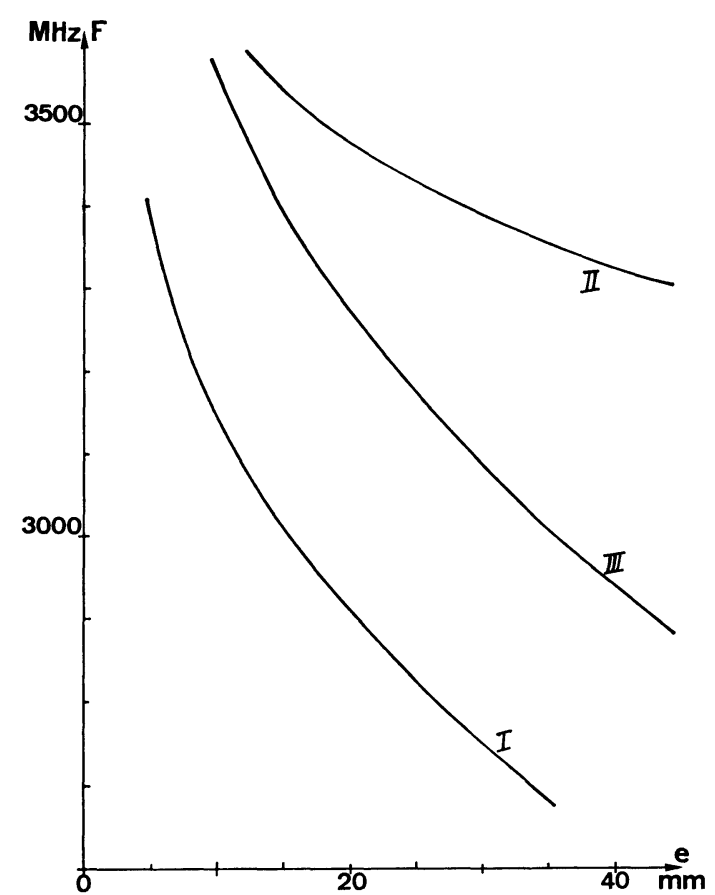

Fig. 6. - Evolution de la fréquence de résonance du mode $\pi$ en fonction de $e$.

[Evolution of the $\pi$-mode resonance frequency versus $e$.]

structure était ouverte dans la direction $\mathrm{O} x$. Le facteur de surtension croît lorsqu'on diminue l'écartement $e$.

Le tableau II donne les valeurs de $Q_{0}$ pour la structure $\mathrm{n}^{\circ} 3$ à auges de section trapézoïdale, munie de plans de court-circuit dans la direction $O x$, en fonction de la distance $D$ de ces plans au bord des auges, pour $e=25 \mathrm{~mm}$.

Dans toutes les mesures, les barres d'écartement, perpendiculaires à $O z$, étaient placées à $d=7,5 \mathrm{~mm}$ des bords des auges. 


\section{TABLEAU I}

$\begin{array}{cccccc}e(\mathrm{~mm}) & 15 & 20 & 25 & 30 & 40 \\ \dot{Q}_{0} & - & - & - & - & - \\ \text { cavité } \mathrm{n}^{\circ} 3\left(v_{\varphi} \simeq c \text { pour le mode } \pi\right) & 1,39 \times 10^{4} & 1,15 \times 10^{4} & 10^{4} & 0,95 \times 10^{4} & 0,89 \times 10^{4}\end{array}$

\section{TABLEAU II}

$\begin{array}{cccccccc}D_{\mathrm{m} \mathrm{m}} & \frac{0}{Q_{0}} & \frac{2}{-} & \frac{7}{-} & \frac{12}{-} & \frac{17}{-} & \frac{22}{27} & \frac{\infty}{10^{4}} \\ 10^{4}\end{array}$

Les valeurs relevées sur le tableau II confirment que $Q_{0}$ croît constamment lorsque $D$ croît. Dans une solution cryogénique, il sera possible de fermer latéralement la cavité à $D \simeq 20 \mathrm{~mm}$ des auges.

4. Mesure de l'impédance shunt $R$ de la structure périodique-détermination des facteurs de qualité $R / Q_{0}$ et $\mathbf{R}_{\text {eff }} / \mathbf{Q}_{0} \cdot-4.1$. MÉTHOde UTILISÉE. - Nous utilisons ici la méthode classique de perturbation du champ électrique par une tige diélectrique placée sur l'axe de la structure périodique transformée en cavité résonnante $[6,7]$. Cette méthode, facile à mettre en œuvre, fournit une perturbation correspondant à la valeur moyenne du carré de l'amplitude du champ électrique le long de la structure. Elle présente en outre l'avantage d'être insensible aux effets d'images dans les parois métalliques lorsque la tige traverse la cavité de part en part $[8,9]$.

On suppose que le rayon de la tige est suffisamment petit pour admettre que le champ reste uniforme le long de sa section droite $A$. Si $\varepsilon \varepsilon_{0}$ désigne la constante diélectrique de la tige, $l$ la longueur de la cavité, $E_{\mathrm{c}}$ l'amplitude du champ électrique à une distance $z$, la perturbation de fréquence de résonance $f$ de la cavité est donnée par l'expression :

$$
\frac{\Delta f}{f}=-\frac{1}{4} \frac{(\varepsilon-1) \varepsilon_{0} A}{W_{\mathrm{c}}} \int_{0}^{l} E_{\mathrm{c}}^{2} \mathrm{~d} z
$$

où $W_{\mathrm{c}}$ est l'énergie totale emmagasinée dans la cavité.

L'impédance shunt est, d'autre part, définie par l'expression suivante :

$$
R=\frac{\left[\int_{0}^{l} E_{\mathrm{c}} \mathrm{d} z\right]^{2}}{P} \cdot \frac{1}{l}
$$

où $P$ est la puissance totale dissipée dans la cavité, liée à la valeur du coefficient de surtension à vide par la relation :

$$
Q_{0}=\frac{\omega W_{\mathrm{c}}}{P}
$$

On en déduit :

$$
R / Q_{0}=\frac{\left[\int_{0}^{l} E_{\mathrm{c}} \mathrm{d} z\right]^{2}}{\omega W_{\mathrm{c}}} \cdot \frac{1}{l}
$$

En éliminant $W_{c}$ entre les expressions (1) et (2), il vient :

$$
\frac{\Delta f}{f}=-\frac{1}{4}(\varepsilon-1) \varepsilon_{0} A \cdot \omega \cdot\left(\frac{R}{Q_{0}}\right) \cdot l \frac{\int_{0}^{l} E_{\mathrm{c}}^{2} \mathrm{~d} z}{\left[\int_{0}^{l} E_{\mathrm{c}} \mathrm{d} z\right]^{2}}
$$

ou encore :

$$
\frac{R}{Q_{0}}=\frac{2}{\pi} \cdot \frac{1}{(\varepsilon-1) \varepsilon_{0} A} \cdot \frac{\Delta f}{f^{2}} \frac{\left[\frac{1}{l} \int_{0}^{l} E_{\mathrm{c}} \mathrm{d} z\right]^{2}}{\frac{1}{l} \int_{0}^{l} E_{\mathrm{c}}^{2} \mathrm{~d} z} .
$$

La quantité $(\varepsilon-1) A$ est obtenue par une mesure annexe d'étalonnage de la tige dans une cavité de référence où le champ électromagnétique est parfaitement connu. Le cas le plus simple est celui de la cavité cylindrique circulaire fonctionnant sur le mode $\mathrm{TM}_{010}$. La perturbation sur l'axe d'une telle cavité résonnant à la fréquence $f_{\mathrm{c}}$ donne un glissement de fréquence $\Delta f_{\mathrm{c}}$, tel que :

$$
\frac{\Delta f_{\mathrm{c}}}{f_{\mathrm{c}}^{2}}=\frac{\pi}{2}(\varepsilon-1) \varepsilon_{0} A \cdot \frac{c \mu_{0}}{a}
$$

où $a$ est le rayon de la cavité de référence ( $a=0,383$ $\left.c / f_{\mathrm{c}}=0,383 \lambda_{\mathrm{c}}\right)$. On en déduit :

$(\varepsilon-1) A=\frac{2}{\pi \varepsilon_{0} \mu_{0}} \cdot \frac{\Delta f_{\mathrm{c}}}{f_{\mathrm{c}}^{2}} \cdot \frac{0,383}{f_{\mathrm{c}}}=\frac{2}{\pi} c^{2} \frac{\Delta f_{\mathrm{c}}}{f_{\mathrm{c}}^{2}} \cdot \frac{0,383}{f_{\mathrm{c}}}$.

En reportant dans (3), il vient, en introduisant l'impédance d'onde du vide :

$$
\begin{gathered}
\eta=\frac{1}{\varepsilon_{0} c}=\mu_{0} c=377 \Omega \\
\frac{R}{Q_{0}}=2,61\left(\frac{\Delta f}{\Delta f_{\mathrm{c}}}\right)\left(\frac{f_{\mathrm{c}}}{f}\right)^{2} \cdot \frac{\left[\frac{1}{l} \int_{0}^{l} E_{\mathrm{c}} \mathrm{d} z\right]^{2}}{\frac{1}{l} \int_{0}^{l} E_{\mathrm{c}}^{2} \mathrm{~d} z} \cdot \frac{\eta}{\lambda_{\mathrm{c}}}
\end{gathered}
$$

Cette expression ne tient évidemment aucun compte du temps de transit des particules accélérées lorsqu'elles traversent la structure. Dans un projet d'accélérateur 
de particules à ondes stationnaires, on prendra comme facteur de mérite l'impédance shunt effective [10]:

$$
\frac{R_{\text {eff }}}{Q_{0}}=\frac{R}{Q_{0}} \cdot \frac{\left[\frac{1}{l} \int_{0}^{l} E_{\mathrm{c}}\left|\cos \frac{\omega}{c} z\right| \mathrm{d} z\right]^{2}}{\left[\frac{1}{l} \int_{0}^{l} E_{\mathrm{c}} \mathrm{d} z\right]^{2}}
$$

ou encore :

$$
\begin{aligned}
\frac{R_{\text {eff }}}{Q_{0}}=2,61\left(\frac{\Delta f}{\Delta f_{\mathrm{c}}}\right) \cdot\left(\frac{f_{\mathrm{c}}}{f}\right)^{2} \times \\
\times \frac{\left[\frac{1}{l} \int_{0}^{l} E_{\mathrm{c}}\left|\cos \frac{\omega}{c} z\right| \mathrm{d} z\right]^{2}}{\frac{1}{l} \int_{0}^{l} E_{\mathrm{c}}^{2} \mathrm{~d} z} \cdot \frac{\eta}{\lambda_{\mathrm{c}}} .
\end{aligned}
$$

Une mesure de la distribution du champ électrique longitudinal sur l'axe à l'aide d'un petit obstacle diélectrique (aiguille ou bille) permet de déterminer le rapport $F$ des valeurs moyennes intervenant dans l'expression $\left(5^{\prime}\right)$.

On notera que dans le cas idéal d'une structure à auges dans laquelle les cellules seraient très faiblement couplées, l'amplitude du champ électrique sur l'axe varierait pratiquement en signaux carrés (cas d'un écartement entre les plaques très faible devant la profondeur des auges) et le facteur $F$ prendrait la valeur :

$$
F=\frac{\left[\frac{1}{l} \int_{0}^{l} E_{\mathrm{c}}\left|\cos \frac{\omega}{c} z\right| \mathrm{d} z\right]^{2}}{\frac{1}{l} \int_{0}^{l} E_{\mathrm{c}}^{2} \mathrm{~d} z}=\left[\frac{\sin \left(\frac{P-t}{P}\right) \frac{\pi}{2}}{\frac{P-t}{P} \frac{\pi}{2}}\right]^{2}
$$

$P$ étant le pas de la structure et $t$ l'épaisseur des parois. Si l'on choisit $P / t=0,1, F=0,488$.

Cette valeur est en fait très voisine de celle que l'on obtient lorsque l'amplitude du champ sur l'axe varie suivant une loi sinusoïdale (cas d'une structure dépourvue d'harmoniques d'espace). Dans ce dernier cas, le calcul donne $F=0,5$. Il apparaît ainsi qu'en première approximation, le facteur $F$ ne dépend pratiquement pas de la distribution du champ axial lorsque $t / P=0,1$, et par conséquent de l'espacement $e$ entre les plaques. Ce résultat n'est valable que lorsque la structure est infiniment longue, c'est-à-dire lorsque le champ se reproduit identique à lui-même d'une auge à l'autre. Dans le cas d'une structure courte où l'on doit tenir compte des effets d'extrémités dus au fait que les plans de court-circuit ne sont pas situés dans les plans de symétrie (cas d'un fonctionnement sur le mode $\pi$ ), la valeur théorique de $F$ (donnée par le $2^{\mathrm{e}}$ nombre de l'expression (6)) doit être multipliée par une expression du type :

$$
F^{\prime}=\frac{1}{N} \frac{\left(\sum_{N} a_{i}\right)^{2}}{\sum_{N} a_{i}^{2}}
$$

où $a_{i}$ représente l'amplitude des champs dans chaque auge et $N$ le nombre total des auges.
4.2 Résultats obtenus. - La figure 7 montre à titre d'exemple la distribution du champ relevé sur

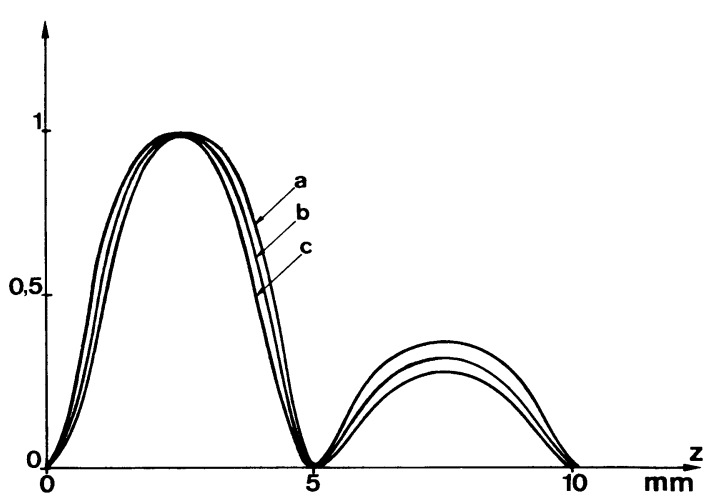

Fig. 7. - Distributions relatives de $E_{z}^{2}(z)$ pour différentes valeurs de $e$ à la fréquence de résonance du mode $\pi$ de la cavité $\mathrm{n}^{\circ} 3$ ( $z=0$ au milieu de la cavité). $a: e=15 \mathrm{~mm}$;

$$
b: e=20 \mathrm{~mm} ; c: e=30 \mathrm{~mm} \text {. }
$$

[Relative $E_{z}^{2}(z)$ distributions for different values of $e$ at $\pi$-mode resonance frequency in cavity $\mathrm{n}^{\circ} 3(z=0$ at the midpoint of the cavity). $a: e=15 \mathrm{~mm} ; b: e=20 \mathrm{~mm} ; c: e=30 \mathrm{~mm}$.

l'une des structures étudiées (structure $\mathrm{n}^{0} 3$ ) pour différentes valeurs de l'écartement $e$. Le calcul graphique du facteur $F$ donne dans tous les cas, une valeur comprise entre 0,45 et 0,46 , en accord avec les prévisions du paragraphe précédent et lorsqu'on tient

\begin{tabular}{|c|c|c|c|c|}
\hline$e(\mathrm{~mm})$ & 15 & 20 & 25 & 30 \\
\hline$f_{\pi}(\mathrm{MHz})$ & 3007 & 2905 & 2815 & 2749 \\
\hline$R / Q(\Omega / \mathrm{cm})$ & 66,2 & 58,1 & 46,9 & 40,5 \\
\hline$R_{\mathrm{eff}} / Q_{0}(\Omega / \mathrm{cm})$ & 30,4 & 26,7 & 21,6 & 18,6 \\
\hline
\end{tabular}
compte des effets de bord.

Le tableau III donne les valeurs de $f_{0}, R / Q_{0}$ et $R_{\text {eff }} / Q_{0}$ pour la structure $\mathrm{n}^{0} 1$ résonnant sur le mode $\pi$ en fonction de l'écartement $e$.

\section{TABLEAU III}

\begin{tabular}{|c|c|c|c|}
\hline$e(\mathrm{~mm})$ & 15 & 20 & 25 \\
\hline$f_{\pi}(\mathrm{MHz})$ & 3522 & 3461 & 3431 \\
\hline$R / Q_{0}(\Omega / \mathrm{cm})$ & 49,5 & 32,9 & 21,1 \\
\hline$R_{\mathrm{eff}} / Q_{0}(\Omega / \mathrm{cm})$ & 22,3 & 14,8 & 9,5 \\
\hline
\end{tabular}

Le tableau IV correspond à la cavité $\mathrm{n}^{0} 2$ résonnant sur le mode $\pi$, utilisable pour accélérer les particules dans la région où $v \simeq c / 2$.

\begin{tabular}{|c|c|c|c|}
\hline$e(\mathrm{~mm})$ & 15 & 20 & 25 \\
\hline$f_{\pi_{2}}(\mathrm{MHz})$ & 3496 & 3389 & 3348 \\
\hline$R / Q_{0}(\Omega / \mathrm{cm})$ & 66,3 & 44,3 & 39,8 \\
\hline$R_{\mathrm{eff}} / Q_{0}(\Omega / \mathrm{cm})$ & 30 & 20 & 18 \\
\hline
\end{tabular}

\section{TABLEAU IV}

Pour la même cavité résonnant sur le mode $\pi / 2$ avec $v \simeq c$, on obtient les résultats donnés sur le tableau $\mathrm{V}$.

\section{TABLEAU V}


TABleau VI

\begin{tabular}{llllllll}
\multicolumn{1}{c}{$(\mathrm{mm})$} & 10 & 15 & 20 & 25 & 30 & 35 & 40 \\
$f_{\pi}(\mathrm{MHz})$ & $\frac{15}{543}$ & $3 \overline{394}$ & $3 \overline{272}$ & $3 \overline{171}$ & 3082 & 3006 & 2938 \\
$R / Q_{0}(\Omega / \mathrm{cm})$ & 87,4 & 72,3 & 60,5 & 51,2 & 42,7 & 39,4 & 29,9 \\
$R_{\text {eff }} / Q_{0}(\Omega / \mathrm{cm})$ & 40,2 & 33,2 & 27,8 & 23,5 & 19,6 & 18,1 & 13,4
\end{tabular}

Enfin, les résultats relatifs au mode $\pi$ de la cavité no 3 à section trapézoïdale à bords arrondis, sont donnés dans le tableau VI.

Pour une cavité parallélépipédique unique résonnant à $3000 \mathrm{MHz}$ sur le mode $\mathrm{TM}_{110}$ et de dimensions $a$, $b, p=\lambda_{0} / 2$, le calcul donne :

$$
\left(R / Q_{0}\right)_{\mathrm{TM}_{110}}=\frac{8 \eta}{\pi \sqrt{a^{2}+b^{2}}},
$$

soit, pour la cellule élémentaire de la structure à auges lorsque $e=0: R / Q_{0}=106,5 \Omega / \mathrm{cm}$ et $R_{\text {eff }} / Q_{0}=52 \Omega / \mathrm{cm}$. C'est la valeur limite que l'on obtient en extrapolant à $e=0$ les valeurs expérimentales données dans le tableau III (Fig. 8).

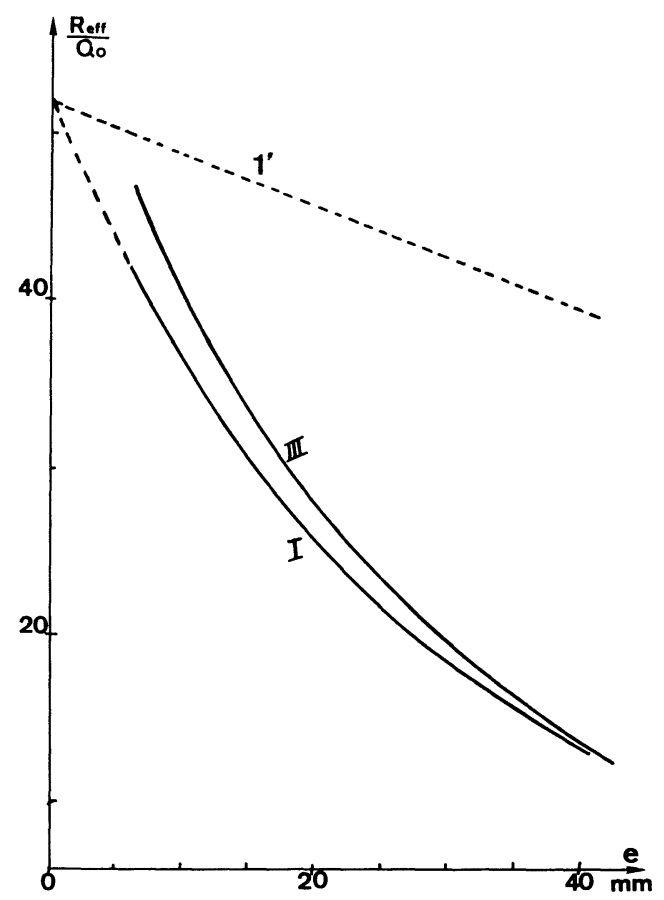

FIG. 8. - Variations de $R_{\text {erf }} / Q_{0}$ en fonction de $e$ (la courbe (1') représente la variation de $R_{\text {eft }} / Q_{0}$ de la cavité parallélépipédique de longueur $p=\lambda_{0} / 2$ fonctionnant sur le mode $\mathrm{TM}_{110}$ ).

$\left[R_{\mathrm{eff}} / Q_{0}\right.$ variations versus $e$ (the curve labelled $\left(1^{\prime}\right)$ represents the $R_{\text {eff }} / Q_{0}$ variation in the closed cavity of length $p=\lambda_{0} / 2$ at the $\mathrm{TM}_{110}$ mode).]

Nos mesures montrent très nettement qu'on aura avantage à utiliser un écartement $e$ aussi faible que possible, compatible avec la section transversale du faisceau, si l'on veut à la fois un facteur $R / Q_{0}$ élevé et une surtension $Q_{0}$ élevée.
En ce qui concerne l'influence des plans de courtcircuit latéraux, nous avons observé une légère augmentation de $R / Q_{0}$ (d'environ $2 \%$ ) lorsqu'on passe de la configuration à $D<20 \mathrm{~mm}$ à celle où $D=\infty$.

L'augmentation de $Q_{0}$, supérieure à $20 \%$, signalée plus haut (tableau II) accompagnée d'un accroissement de $R / Q_{0}$, montre que la structure à champs évanescents suivant $O x$ est supérieure à la structure fermée au niveau des flancs des auges $(D=0)$

Notons enfin qu'un calcul récent $\left({ }^{1}\right)$ par la méthode des mailles appliquée au modèle de la cavité $\mathrm{n}^{0} 3$ courtcircuitée à $D=0$, donne pour $e=25 \mathrm{~mm}$, $R_{\text {eff }} / Q_{0}=22,5 \Omega / \mathrm{cm}$, valeur en excellent accord avec celle obtenue expérimentalement.

5. Mesure des rapports entre les champs électrique et magnétique maximums sur les parois au champ accélérateur sur l'axe. - 5.1. INTRODUCTION. - La valeur du champ accélérateur sur l'axe, dans une structure fonctionnant à la température ambiante, est limitée par les claquages se produisant aux points où le champ électrique atteint sa valeur maximum. Dans une structure utilisant un matériau supraconducteur apparaît une autre limitation, due au champ magnétique hyperfréquence, $B^{\mathrm{HF}}$ qui ne peut dépasser une certaine valeur critique $B_{\mathrm{c}}^{\mathrm{HF}}$, qui est théoriquement et pratiquement égale au champ magnétique critique thermodynamique $B_{\mathrm{c}}$, dans des matériaux de type I $(\mathrm{Pb}, \mathrm{Sn})$. Dans le cas de matériaux supraconducteurs de type II ( $\mathrm{Nb}$ ou $\left.\mathrm{Nb}_{3} \mathrm{Sn}\right)$, on a obtenu des valeurs $B_{\mathrm{c}}^{\mathrm{HF}}$ supérieures à $B_{\mathrm{c}_{1}}$ (1 $1^{\mathrm{er}}$ champ critique) et des recherches sont actuellement menées pour vérifier si la limite supérieure de $B_{\mathrm{c}}^{\mathrm{HF}}$ est ici encore égale à $B_{\mathrm{c}}$.

Il est donc important de connaître les rapports $E_{\mathrm{p}} / E_{\text {eff }}$ et $B_{\mathrm{p}} / E_{\text {eff }}, E_{\mathrm{p}}$ et $B_{\mathrm{p}}$ désignant les valeurs maximums de $E$ et $B$ existant sur les parois, pour la valeur du champ accélérateur effectif :

$$
E_{\text {eff }}=\frac{1}{l} \int_{0}^{l} E_{\mathrm{c}}\left|\cos \frac{\omega}{c} z\right| \mathrm{d} z
$$

Ces rapports ont, à nouveau, été mesurés en mettant en œuvre la méthode des perturbations.

5.2. MÉTHOde DE MESURE DES CHAMPS ÉLeCTRIQUES ET MAGNÉTIQUES SUR LES PAROIS. - Nous avons utilisé la méthode décrite par Bernard et coll. [11] et Vaguine [12] qui ont montré que la forme la plus adéquate pour l'objet perturbateur est une demi-sphère

(1) Weingarten, W., Communication privée. 
diélectrique ou métallique que l'on déplace sur la paroi de la structure dans la zone de champ électrique ou magnétique maximum. L'obstacle est porté par un fil de nylon très fin $(2 / 10 \mathrm{~mm})$ qui le traverse suivant une corde $\left({ }^{2}\right)$. Dans le cas de mesures du champ magnétique à l'aide de l'obstacle métallique, on a collé sur la demi-sphère une feuille de mylar $(6 \mu)$ pour éviter le contact métallique avec la surface à mesurer.

Comme dans le cas de la mesure de l'impédance shunt à l'aide d'une tige diélectrique, ces obstacles ont été calibrés dans une cavité de référence $\mathrm{TM}_{010}$. La demi-sphère métallique a été placée contre la paroi latérale où le champ magnétique est uniforme et le champ électrique nul. La demi-sphère diélectrique a été placée au centre du fond de la cavité où le champ électrique axial est pratiquement uniforme.

Afin de rapporter les valeurs des champs électriques et magnétiques maximums au champ accélérateur effectif, nous avons mis à profit les mesures précédentes effectuées à l'aide d'une tige diélectrique et celles permettant de déterminer le facteur $F$. On obtient ainsi :

$$
\frac{E_{\mathrm{p}}}{E_{\mathrm{eff}}}=\sqrt{\frac{\delta f_{1}}{\delta f_{\mathrm{c}_{1}}} \frac{\Delta f_{\mathrm{c}}}{\Delta f} \frac{L}{L_{\mathrm{c}}} F}
$$

et

$$
\frac{B_{\mathrm{p}}}{E_{\text {eff }}}=\sqrt{\frac{\delta f_{2}}{\delta f_{\mathrm{c}_{2}}} \frac{\Delta f_{\mathrm{c}}}{\Delta f} \frac{L}{L_{\mathrm{c}}} F} \cdot \frac{B_{0}}{E_{0}}
$$

où $\delta f_{1}$ et $\delta f_{2}$ désignent respectivement les perturbations de fréquence par la demi-bille diélectrique ou métallique, $\delta f_{\mathrm{c}_{1}}$ et $\delta f_{\mathrm{c}_{2}}$ les perturbations correspondantes dans la cavité de référence, $\Delta f$ et $\Delta f_{\mathrm{c}}$ les perturbations créées par la tige diélectrique sur l'axe, $L$ et $L_{\mathrm{c}}$ les longueurs de la cavité à étudier et de la cavité de référence, $B_{0} / E_{0}$ le rapport du champ magnétique sur la paroi au champ électrique sur l'axe dans la cavité de référence :

$$
B_{0} / E_{0}=\frac{0,52 \mu_{0}}{\eta}=17,3 \mathrm{G} / \mathrm{MV} \mathrm{m}^{-1}
$$

Les variations de fréquence $\delta f_{i}$ à mesurer avec un obstacle de très petite taille étant très faibles (de l'ordre de $20 \mathrm{kHz}$ ), nous avons utilisé le dispositif représenté sur la figure 9 dans lequel la fréquence du générateur Gunn est à chaque instant verrouillée sur la fréquence de résonance $f$ de la cavité grâce à un signal d'erreur fourni par un mélangeur équilibré. Un compteur calculateur fournit une tension proportionnelle aux écarts de fréquences $\delta f_{i}$, qui est envoyée sur une table traçante XY dont l'autre entrée est commandée par un signal d'amplitude proportionnelle aux déplacements de l'obstacle.

(2) Ces obstacles perturbateurs, de diamètre $\varphi=2,5 \mathrm{~mm}$, manufacturés en Suisse, nous ont été aimablement fournis par H. Lengeler, actuellement au Centre de Physique Nucléaire de Karlsruhe (R. F. A.), que nous remercions vivement.

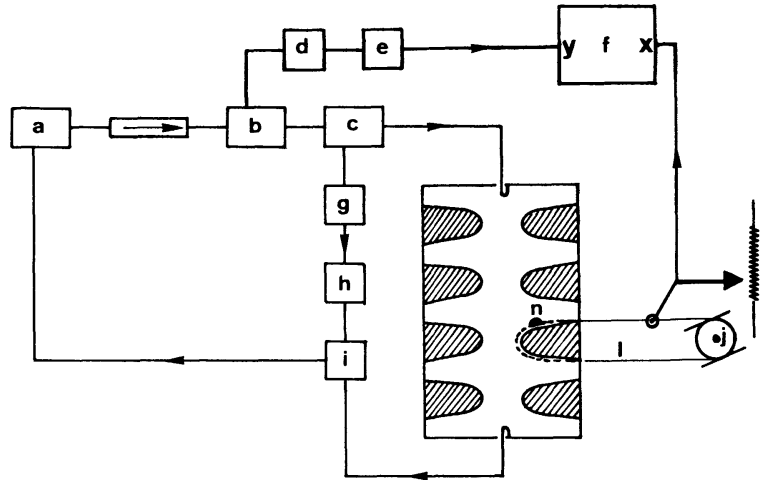

Fig. 9. - Dispositif expérimental de mesure des perturbations de fréquences de résonance par des obstacles diélectriques ou métalliques : $a$ : oscillateur à diode Gunn; $b$ et $c$ : coupleurs directifs ; $d$ : compteur de fréquence ; $e$ : convertisseur analogique ; $f$ : table traçante $\mathrm{XY} ; g$ et $h$ : déphaseur et atténuateur variables ; $i$ : mélangeur équilibré ; $j:$ moteur d'entraînement ; $l:$ fil de nylon; $n:$ obstacle perturbateur.

[Experimental setup for the precise measurement of frequency perturbations produced by small dielectric or metallic objects : $a$ : Gunn diode oscillator; $b$ and $c$ : directional couplers; $d$ : frequency counter ; $e$ : analog converter $f: \mathrm{XY}$ recorder ; $g$ and $h$ : variable attenuator and phase shifter ; $i$ : balanced mixer ; $j$ : pulling motor; $l$ : nylon string; $n$ : perturbing object.]

La stabilité de fréquence obtenue est d'environ $\pm 40 \mathrm{~Hz}$ pour des périodes de 30 secondes nécessaires au passage des obstacles perturbateurs dans la cavité. Le champ électrique a été mesuré dans la zone

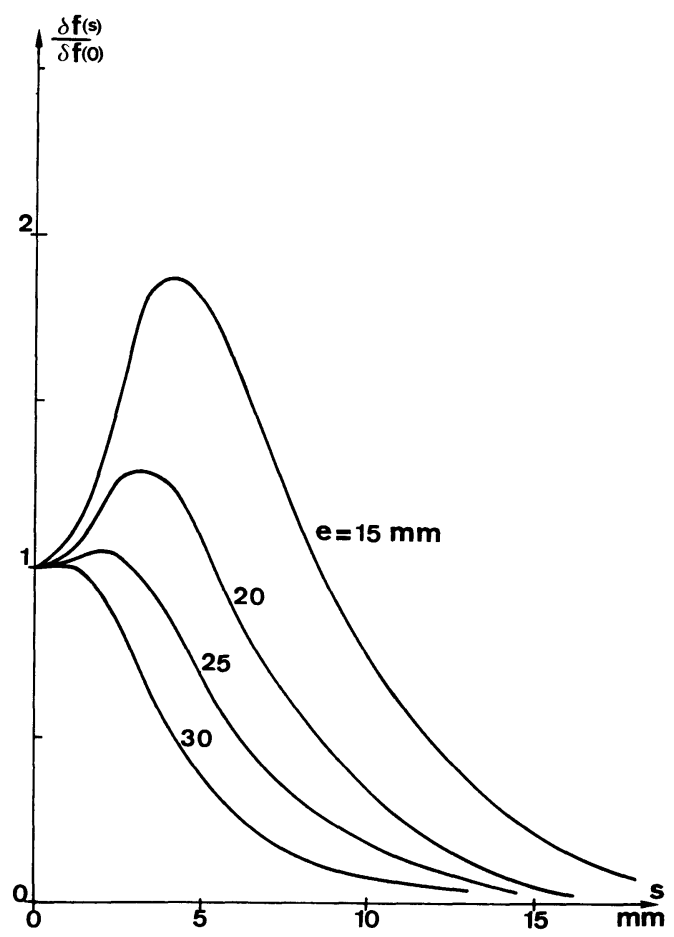

FIG. 10. - Variation relative $\delta f(s) / \delta f(0)$ pour différentes valeurs de $e$.

[Relative variation of $\delta f(s) / \delta f(0)$ for different values of $e$.] 
d'intersection des parois séparant les auges avec le plan y $\mathrm{O} z$ (Fig. 1). La principale difficulté de la méthode réside ici dans le fait que le champ électrique peut présenter de fortes inhomogénéités, notamment dans le cas des faibles espacements $e$, qui correspond à un faible couplage entre les auges. La figure 10 montre la variation relative $\delta f(s) / \delta f(0), s$ désignant l'abscisse curviligne comptée sur le contour de la paroi, en fonction de $e$. On a reporté sur la figure 11 l'évolution de la

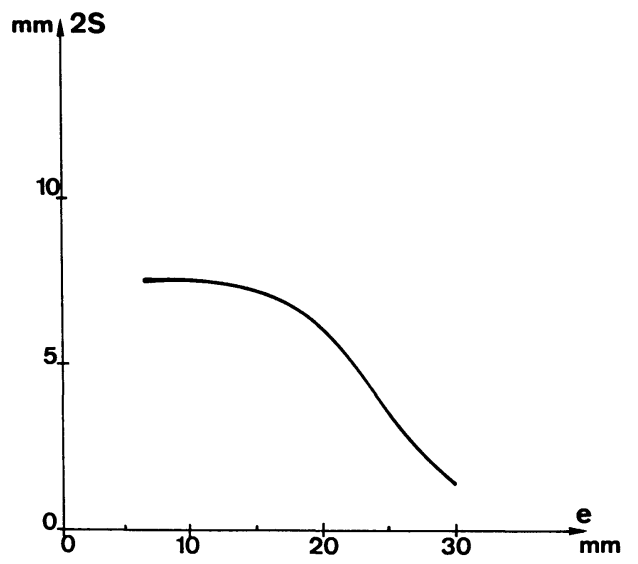

FIG. 11. - Evolution de la position du maximum du champ électrique en fonction de $e$.

[Evolution of the maximum electric field position versus $e$.]

position du maximum du champ électrique en fonction de $e$. (La connaissance de cette position serait nécessaire dans un calcul de trajectoires d'électrons produits par émission de champ).

En ce qui concerne le champ magnétique $B_{\mathrm{p}}$, les mesures ont été effectuées sur le fond des auges dans une région où le champ électrique est nul. (Dans une cavité rectangulaire fermée résonnant sur le mode $\mathrm{TM}_{110}, B_{\mathrm{p}}$ est maximum au milieu de la paroi normale à l'axe $\mathrm{Oy}$ ). Ces mesures ont été complétées, dans le cas de la cavité $\mathrm{n}^{0} 3$, par la détermination du champ $B_{\mathrm{p}_{\mathrm{a}}}$ sur l'arrondi du bord d'auge parallèle au plan $y \mathrm{O} z$ (Fig. 1).

5.3. RÉSUltats obTenUS. - Les tableaux VII, VIII, IX et $\mathrm{X}$ donnent les valeurs des 3 rapports des champs définis précédemment en fonction de l'épaisseur $e$ pour les trois structures étudiées.

\section{TABLEAU VII}

Cavité $n^{0} 1$ à 6 auges. Mode $\pi$

\begin{tabular}{lcccc}
\multicolumn{1}{c}{$e(\mathrm{~mm})$} & 15 & 20 & 25 & 30 \\
$E_{\mathrm{p}} / E_{\mathrm{z}_{\max }}$ & - & - & - & - \\
$E_{\mathrm{p}} / E_{\text {eff }}$ & 1,59 & 1,67 & 1,75 & 1,97 \\
$B_{\mathrm{p}} / E_{\text {eff }}(\mathrm{G} / \mathrm{MV} / \mathrm{m})$ & 4 & 4,4 & 4,8 & 5,3 \\
& 72 & 70 & 71 & 73
\end{tabular}

TABLEAU VIII

Cavité $n^{\circ} 2$ à 4 auges. Mode $\pi / 2\left(v_{\varphi} \simeq c\right)$

\begin{tabular}{llll}
\multicolumn{1}{c}{$e(\mathrm{~mm})$} & 15 & 20 & 25 \\
$E_{\mathrm{p}} / E_{\mathrm{z}_{\max }}$ & - & - & - \\
$E_{\mathrm{p}} / E_{\text {eff }}$ & 1,76 & 1,88 & 2,02 \\
$B_{\mathrm{p}} / E_{\text {eff }}(\mathrm{G} / \mathrm{MV} / \mathrm{m})$ & 3,4 & 4,4 & 4,9 \\
& 68 & 66 & 67
\end{tabular}

\section{TABLEAU IX}

Cavité $n^{\circ} 2$ à 4 auges. Mode $\pi\left(v_{\varphi} \simeq c / 2\right)$

\begin{tabular}{llll}
\multicolumn{1}{c}{$e(\mathrm{~mm})$} & 15 & 20 & 25 \\
$E_{\mathrm{p}} / E_{\mathrm{z}_{\max }}$ & - & - & - \\
$E_{\mathrm{p}} / E_{\text {eff }}$ & 1,33 & 1,38 & 1,56 \\
$B_{\mathrm{p}} / E_{\text {eff }}(\mathrm{G} / \mathrm{MV} / \mathrm{m})$ & 3,4 & 3,6 & 3,7 \\
& 69 & 66 & 66
\end{tabular}

\section{TABleau $X$}

Cavité $n^{0} 3$ à 4 auges. Mode $\pi\left(v_{\varphi} \simeq c\right)$.

$\begin{array}{cccccccc}e(\mathrm{~mm}) & 10 & 15 & 20 & 25 & 30 & 35 & 40 \\ E_{\mathrm{p}} / E_{\mathrm{z}_{\max }} & 1,06 & 1,3 & 1,37 & 1,43 & 1,52 & 1,78 & 2,1 \\ E_{\mathrm{p}} / E_{\text {eff }} & 2,4 & 2,7 & 2,95 & 3,4 & 3,7 & 4,6 & 6,4 \\ B_{\mathrm{p}} / E_{\text {eff }} & & & & & & & \\ (\mathrm{G} / \mathrm{MV} / \mathrm{m}) & & 57 & 60 & 63,5 & 68 & 69 & \\ B_{\mathrm{p}_{\mathrm{a}}} / E_{\text {eff }} & & & & & & & \\ (\mathrm{G} / \mathrm{MV} / \mathrm{m}) & & 60 & & 80,3 & & 103 & \end{array}$

L'examen de ces résultats montre la supériorité de la cavité no 3 sur les autres structures, plus conventionnelles.

Des mesures complémentaires, effectuées sur cette structure court-circuitée latéralement $(0<D<27)$ ont montré qu'aux erreurs expérimentales près, les rapports des champs étudiés restent constants.

Le tableau suivant fournit la comparaison entre les résultats expérimentaux et théoriques sur cette cavité à auges trapézoïdales à bords arrondis.

\section{TABlEAU XI}

Comparaison des résultats expérimentaux et théoriques sur la cavité à auges trapézoïdales à bords arrondis $e=25 \mathrm{~mm}$.

$\begin{array}{lccc} & & \text { (Wupperthal) } & \\ R_{\text {eff }} / Q_{0} & 23,5 & 22,5 & 4 \% \\ f_{\pi} & 3169 & 3055 & 3,6 \% \\ E_{\mathrm{p}} / E_{\text {eff }} & 3,4 & 3,9 & 14 \% \\ B_{\mathrm{p}_{\mathrm{a}}} / E_{\text {eff }}(\mathrm{G} / \mathrm{MV} / \mathrm{m}) & 80,3 & 84 & 4,4 \% \\ B / E_{\text {eff }}(\mathrm{G} / \mathrm{MV} / \mathrm{m}) & 63,5 & 59 & 7 \%\end{array}$

L'écart le plus important entre la théorie et l'expérience porte sur la valeur de $E_{\mathrm{p}} / E_{\text {eff }}$. Cet écart peut s'expliquer par le fait que la mesure est effectuée, comme nous l'avons déjà indiqué, dans une région où 
le champ est, en général, fortement inhomogène (cf. Fig. 10). La demi-bille métallique de $2,5 \mathrm{~mm}$ de diamètre donne une valeur moyenne autour du maximum du champ et conduit à une valeur optimiste du rapport $E_{\mathrm{p}} / E_{\text {eff }}$. Un raffinement de la méthode expérimentale consisterait à mesurer le rapport $E_{\mathrm{p}} / E_{\text {eff }}$ en utilisant un jeu de demi-billes de diamètres différents et à extrapoler les résultats obtenus dans le cas d'objets infiniment petits [12].

6. Conclusion. - La structure à auges possède des propriétés intéressantes qui permettent d'envisager son utilisation dans un accélérateur supraconducteur, avec l'avantage, par rapport au guide à iris circulaire classique :

- d'une réalisation plus économique ;

- de l'absence de soudures entre les cellules, sources de dégradation des performances à l'état supraconducteur.

La comparaison des valeurs des paramètres de cette structure, optimisée pour un écartement des plaques égal à $15 \mathrm{~mm},\left(R / Q_{0}=33,2 \Omega / \mathrm{cm} ; E_{\mathrm{p}} / E_{\text {eff }}=2,7\right.$; $\left.B_{\mathrm{p}} / E_{\text {eff }}=57 \mathrm{G} / \mathrm{MV} / \mathrm{m}\right)$, aux valeurs correspondantes d'une structure - celle du MIT [13] — utilisant un guide à iris en ondes stationnaires $\left(R / Q_{0}=25 \Omega / \mathrm{cm}\right.$; $\left.E_{\mathrm{p}} / E_{\text {eff }}=3,3 ; B_{\mathrm{p}} / E_{\text {eff }}=4,5 \mathrm{G} / \mathrm{MV} / \mathrm{m}\right)$ montre, en outre, la compétitivité de la première.

Une structure de ce type en niobium sera prochainement réalisée et testée à fort champ pour déterminer la valeur limite du champ accélérateur $E_{\text {eff }}$ imposée par les claquages électrique ou magnétique.

Remerciements. - Les auteurs remercient vivement les chercheurs de l'équipe du Professeur Piel de l'Université de Wupperthal (RFA) et tout particulièrement le Dr. W. Weingarten, qui a bien voulu s'intéresser au problème de ces structures à auges et a effectué le calcul des paramètres caractéristiques de la cavité $\mathrm{n}^{\circ} 3$. Ils remercient également $M$. Mazubert qui a réalisé le dispositif de stabilisation de fréquence et a effectué l'ensemble des mesures sur ces structures.

\section{Bibliographie}

[1] Sundelin, R., Borstel, E., KirChgessner, I., Rice, D., TIGNER, M., « $3 \mathrm{GHz}$ superconducting accelerator cavity for use in an electron synchrotron », IEEE Trans. NS-20 (1973) 98.

[2] Sundelin, R., Kirchgessner, I., Padamsee, H., Philips, H. L., Rice, D., Tigner, M., v Borstel, E., « Application of superconducting $\mathrm{rf}$ accelerating sections to an electron synchrotron. A progress report", Proc. Ninth Int. Conf. High Energy Acc., Stanford (SLAC, 1974) p. 128.

[3] Kirchgessner, I., Padamsee, H., Philips, H. L., Rice, D., Sundelin, R., Tigner, M., v Borstel, E., «Superconducting cavities for synchrotron use », IEEE Trans. NS-22 (1975) p. 1141.

[4] BAUER, W., "Uberlegungen zur konstruktion einer 700 $\mathrm{MHz}$ Waffeleisen struktur », Institut für Experimentelle Kernphysik, IEKP II/III, Interner Bericht 76151-LIN (mars 1976).

[5] Boussoukaya, M., NguYen Tuong Viet, Septier, A., «Structure accélératrice de symétrie plane à auges rectangulaires », J. Physique Lett., 37 (1976) 317.

[6] Gallagher, W. J., " Mesure de l'impédance shunt des structures périodiques ", Onde Electr., n ${ }^{\circ} 407$ (février 1961) p. 200.
[7] ZYNGIER, H., «L'impédance shunt des cavités radiofréquences ", Rap. interne de l'Accélérateur Linéaire d'Orsay, LAL 25 (juillet 1962).

[8] Waldron, R. A., "Perturbation theory of resonant cavities ", Proc. IEEE (London), 107 C 272.

[9] HahN, H., Halama, H. J., « Perturbation measurement of transverse $\mathrm{R} / \mathrm{Q}$ in iris loaded waveguides ", IEEE Trans. MTT-16 (1968) 20.

[10] Dome, G., « Review and survey of accelerating structures ", Linear Accelerators, P. Lapostolle et A. Septier, (North Holland) 1970 p. 637.

[11] Bernard, P., Lengeler, H., Vaguine, V., « Results of peak field measurements for uniform- and bi-periodic deflecting cavities », J. Appl. Phys. 40 (1969) 4989.

[12] VaGuine, V., « Etude des ondes électromagnétiques hybrides dans les structures cylindriques. Application des résultats aux modes déflecteurs ", Thèse de Doctorat d'Etat, Orsay (17 décembre 1970); $\mathrm{n}^{\circ}$ d'ordre : 729 ; enregistrée au C. N. R. S. sous le $\mathrm{n}^{\circ}$ AO 5227.

[13] Loew, G., Neal, R. B., "Review and survey of accelerating structures », Linear Accelerators, P. Lapostolle et A. Septier (North Holland) 1970 p. 39. 